\title{
An Alternative Aspirin Desensitization Protocol for Patients with Aspirin- Exacerbated Respiratory Disease
}

\author{
Funda Aksu ${ }^{1}$ and Kurtuluş Aksu ${ }^{2^{*}}$ \\ ${ }^{1}$ Department of Chest Diseases, Hacettepe University Faculty of Medicine,Ankara, Turkey. \\ ${ }^{2}$ Department of Chest Diseases, Division of Immunology and Allergy, Ankara Atatürk Chest Diseases and Chest Surgery Hospital, Ankara, Turkey.
}

*Corresponding author: Aksu K, Department of Chest Diseases, Division of Immunology and Allergy, Ankara Atatürk Chest Diseases and Chest Surgery Hospital, Ankara, Turkey; Email: kurtulusaksu@yahoo.com

Received date: May 31, 2015; Accepted date: June 19, 2015; Published date: June 26, 2015

Copyright: (c) 2015 Aksu K, et al. This is an open-access article distributed under the terms of the Creative Commons Attribution License, which permits unrestricted use, distribution, and reproduction in any medium, provided the original author and source are credited.

\section{Letter}

Although the article entitled "An Alternative Aspirin Desensitization Protocol for Patients with Aspirin-Exacerbated Respiratory Disease" is an interesting paper promising an alternative method for ASA desensitization, it fails to conclude in such a method. This is firstly because the authors could not reach the maximum dosage in about half of the study population, and secondly the study design is not based on any rationale to shift into an alternative protocol. A FEV1 decrease by $20 \%$ in the standard protocol, in this study, is defined as 'failure to reach desensitization during classical short term ASA desensitization protocol' and is accepted as an indication to shift into long term desensitization protocol that they offer. However, in the ASA desensitization protocol widely practised by most of the allergy clinics [1] the same symptom provoking dose is repeated the same or the other day if patient comes across such a FEV1 decrease. With this, mostly the reaction does not reoccur and the symptoms resolve either spontaneously or by giving appropriate treatment [2]. So with this methodology the research fails to reveal any superiority of the long-course alternative protocol to the classical one. In fact the title of the manuscript promises an interesting paper and would be so if the authors had shown a valid protocol for patients that have really failed desensitization protocol upon having a severe anaphylactic reaction or serial reactions with the same dose of ASA restricting any dose increment. Shifting into alternative protocol based on election of the patient after experiencing a mild reduction in FEV1 or nasopharyngeal/periorbital symptoms is another irrelevant clinical practice in aspirin desensitization. Because a desensitization protocol completed without any reaction is not possible.

Moreover, the protocol has no superiority to the classical shortterm protocol in establishing the effective dose in all patients. The authors concluded that $300 \mathrm{mg} /$ day ASA might be effective instead of higher doses but they do not mention any related clinical information to validate this conclusion.

Lastly, no clear protocol is offered in the paper because there is no standard dose increment for one initiation dose when the tables are examined.

\section{References}

1. Lee RU, Stevenson DD (2011) Aspirin-exacerbated respiratory disease: evaluation and management. Allergy Asthma Immunol Res 3: 3-10.

2. Aksu K, Kurt E, Alatas Ö, Gülbas Z (2014) Effect of aspirin desensitization on $\mathrm{T}$-cell cytokines and plasma lipoxins in aspirinexacerbated respiratory disease. Allergy Asthma Proc 35: 148-155. 